\title{
A Prática da Avaliação Psicológica em Neuropsicologia e Psicologia do Trânsito Durante Estágio de Formação Profissional
}

\author{
The Practice of Psychological Assessment in Neuropsychology and Traffic Psychology \\ During Professional Internship
}

\section{La Práctica de la Evaluación Psicológica en Neuropsicología y Psicología del Tránsito Durante la Etapa de Formación Profesional}

\author{
Jeanny Joana Rodrigues Alves de Santana ${ }^{1}$ \\ Universidade Federal de Uberlândia \\ Marina Celestino Soares \\ Faculdade Pitágoras
}

\begin{abstract}
Resumo
O objetivo do estudo foi discutir a prática da avaliação psicológica nas áreas da neuropsicologia e psicologia do trânsito a partir de relato de experiência de estágio nestas áreas. Cinco estagiários entrevistaram psicólogos nos campos, acompanharam processos de avaliação psicológica e redigiram relatório de atividades. Estes registros foram sintetizados na forma de levantamento de situações-problema da atuação profissional nos contextos e discutidos qualitativamente com base na literatura da área. Os participantes consideraram importante o debate sobre a prática baseada em evidências e o desenvolvimento científico de constructos em psicologia e de novas tecnologias de avaliação psicológica. O estágio em avaliação psicológica em neuropsicologia e psicologia do trânsito foi oportunidade factível para análise da formação competente e ética em avaliação psicológica, comprometida com o desenvolvimento da psicologia como ciência e profissão.
\end{abstract}

Palavras-chave: avaliação psicológica, prática, pesquisa, neuropsicologia, psicologia do trânsito

\section{Abstract}

The aim of the study was to discuss the practice of psychological assessment in the areas of neuropsychology and traffic psychology from internship experience reports in these areas. Five trainees interviewed psychologists in locus, participated of psychological evaluation processes, and drafted an activity report. These records were synthesized in the form of a survey of problem situations of the professional performance in the contexts and discussed qualitatively based on the literature of the area. Participants considered the importance of debate on evidence-based practice and the scientific development of constructs in psychology and new technologies of psychological assessment. The internship in psychological assessment in neuropsychology and traffic psychology was a feasible opportunity for analysis of competent and ethical training in psychological assessment, committed to the development of psychology as a science and profession.

Keywords: psychological assessment, practice, science, neuropsychology, psychology of traffic

\section{Resumen}

El objetivo del estudio fue analizar la práctica de la evaluación psicológica en las áreas de neuropsicología y psicología del tráfico a partir de los informes de experiencia en prácticas en estas áreas. Cinco aprendices entrevistaron a psicólogos en el locus, participaron en procesos de evaluación psicológica y redactaron un informe de actividad. Estos registros fueron sintetizados en forma de una encuesta de situaciones problemáticas del desempeño profesional en los contextos y discutidos cualitativamente en base a la literatura del área. Los participantes consideraron la importancia del debate sobre la práctica basada en la evidencia y el desarrollo científico de construcciones en psicología y nuevas tecnologías de evaluación psicológica. La pasantía en evaluación psicológica en neuropsicología y psicología del tráfico fue una oportunidad viable para el análisis de una formación competente y ética en la evaluación psicológica, comprometida con el desarrollo de la psicología como ciencia y profesión.

Palabras-clave: evaluación psicológica, práctica, ciencia, neuropsicología, psicología del tránsito

\footnotetext{
${ }^{1}$ Endereço de contato: Universidade Federal de Uberlândia - Instituto de Psicologia (IPUFU), Campus Umuarama - Bloco 2C - Sala 34 Av. Pará, 1720 - Bairro Umuarama, Uberlândia, MG - CEP 38400-902. E-mail: jeannysantana@yahoo.com.br
} 


\section{Introdução}

A avaliação psicológica é o processo técnico-científico de coleta e integração de dados sobre fenômenos psicológicos e é feita por meio de instrumentos como entrevistas, testes e observações do comportamento. Esta área exige qualificação específica, voltada para o desenvolvimento de conhecimentos e habilidades para selecionar os instrumentos, organizar a coleta de informações e integrar os dados de modo a elaborar conclusões seguras sobre o fenômeno que está sendo avaliado (Groth-Marnart \& Wright, 2016). Além disto, o psicólogo tem obrigações no decorrer do processo, como, por exemplo, seguir as diretrizes éticas que indicam em que situações os testes devem ser administrados, como armazenar os dados coletados de modo seguro e de que forma os resultados devem ser sintetizados e apresentados aos clientes (Cohen, Swerdlik, \& Sturman, 2014).

A neuropsicologia e a psicologia do trânsito são duas áreas de atuação profissional que têm em comum o fato de serem fundamentadas em conhecimentos teóricos e técnicos da avaliação psicológica. A primeira tem como objetivo geral identificar padrões de desempenho em um conjunto de domínios de funcionamento cognitivo e comportamental, que tenham significado para a vida do paciente em um quadro de lesões cerebrais ou de déficits neurocognitivos (Casas, Calamia, \& Tranel, 2018). A segunda tem como finalidade ampla a investigação do perfil de funções psicológicas com implicações para o contexto específico do trânsito (Conselho Nacional de Trânsito, 2012).

A ligação que se estabelece no presente trabalho entre as duas áreas se dá não somente em função da experiência das autoras, mas, principalmente, pela importância desta relação para a psicologia científica e aplicada. Em um panorama geral, é possível mencionar que os conhecimentos da neuropsicologia são muito utilizados no contexto do trânsito. Pesquisadores e profissionais buscam identificar o impacto de variáveis neurocognitivas, como por exemplo, as doenças cerebrais e o envelhecimento na aptidão para dirigir. Muito é investido no desenvolvimento de procedimentos capazes de fornecer predição do comportamento do indivíduo no trânsito, a partir dos resultados de testes do perfil cognitivo e de características da personalidade (Brown et al., 2017). A prevenção de acidentes de trânsito depende da identificação de grupos de risco. As falhas no desenvolvimento neurocognitivo de adultos jovens, principalmente no que tange às funções executivas, podem contribuir para o alto índice de acidentes com mortes nesta faixa etária (White, Young, \& Rakotonirainy, 2012).

Alguns desafios comuns da formação profissional em psicologia do trânsito e neuropsicologia também são tratados neste estudo. Os limites da competência profissional dependem do desenvolvimento de conhecimentos, habilidades e atitudes "de forma a garantir tanto o domínio de instrumentos e estratégias de avaliação e de intervenção quanto a competência para selecioná-los, avaliá-los e adequá-los a problemas e contextos específicos de investigação e ação profissional" (Ministério da Educação, 2011).

Os guias de referência para o ensino da avaliação psicológica nas universidades sugerem que as dimensões teóricas e práticas dos cursos sejam articuladas para promover uma formação menos tecnicista e mais crítica com questões como ética e utilidade do processo avaliativo (Noronha, Castro, Ottati, Barros, \& Santana, 2013; Nunes et al., 2012; Borsa, 2016; Castro, 2013; Eisman \& Nordal, 2017). De relevância para as áreas da neuropsicologia e 
psicologia do trânsito é a recomendação para que o ensino da avaliação psicológica ajude o profissional a apresentar com clareza os custos-benefícios de seu trabalho e seja capaz de oferecer soluções potenciais para o cuidado integral em saúde (Wit, Souza, \& Cruz, 2016; Eisman \& Nordal, 2017). O compartilhamento de experiências de formação profissional em avaliação psicológica pode ser estratégia de debate dos pontos nodais neste campo e esclarecimento acerca da relação teoria-prática de formação nas áreas mencionadas.

Este estudo tem como objetivo principal discutir a prática da avaliação psicológica nas áreas da neuropsicologia e psicologia do trânsito a partir de relato de experiência de estágio nestas áreas. A explanação foi estruturada considerando a contextualização do trabalho realizado, seguida por levantamento de temas e situações-problema da atuação em avaliação psicológica abordados durante a prática.

\section{O Contexto do Estágio Realizado em Neuropsicologia e Psicologia do Trânsito}

Os cinco estudantes de psicologia de uma universidade pública, que na ocasião cursavam o quinto período da graduação, realizaram estágio em uma clínica de avaliação de candidatos à obtenção de carteira de motorista e em uma clínica particular de avaliação neuropsicológica. Foram atividades desenvolvidas nestes contextos: entrevistas com as profissionais responsáveis pelos atendimentos; registro de observações sistemáticas de comportamentos (sessões de aplicação de testes; etapas de correção e elaboração de relatórios; sessões de devolutivas de avaliações); participação em reuniões de discussão de casos; debates teórico-práticos em reuniões com a professora orientadora e elaboração de relatórios de estágio.

Os relatórios do estágio deveriam conter uma narrativa dos casos discutidos sob supervisão, contendo (1) Encaminhamento: motivos, queixas, quem solicitou, sintomas, hipótese inicial; (2) Contrato de trabalho: duração do processo; quantidade de sessões; responsabilidades; (3) Método (instrumentos; procedimento de coleta de dados; método de análise dos dados); (4) Resultados (síntese dos resultados da avaliação: entrevistas, testes, levantamento da história clínica, interpretação dos dados; outras técnicas); (5) Discussão (diagnóstico; prognóstico; comunicação dos resultados; laudo). Considerando a abrangência teórico-prática destes relatórios de estágio, eles foram utilizados para extração de informações para a realização do presente trabalho. A análise descritiva destas narrativas suscitou o levantamento de situações-problema vinculadas à prática realizada, e indicadas como aspectos importantes para a formação profissional experienciada. Estas questões foram debatidas no presente estudo a partir da fundamentação teórica da avaliação psicológica. Considerando a necessidade de manter o sigilo dos participantes, seus nomes foram substituídos pelos códigos P1, P2, E1, E2 e E3, sendo P1 designando a psicóloga do campo de estágio em psicologia do trânsito; P2 a psicóloga do campo de estágio em neuropsicologia; E1, E2 e E3 a designação de alguns dos estagiários que participaram da experiência.

As temáticas identificadas no estudo (Figura 1) serão apresentadas nos seguintes tópicos: (1) o problema do uso de testes psicológicos para diferentes contextos e populações; (2) habilidades profissionais para atuação em avaliação psicológica: o especialista ou o generalista? e (3) a atuação em avaliação psicológica e as questões sócio-políticas e econômicas da profissão. 

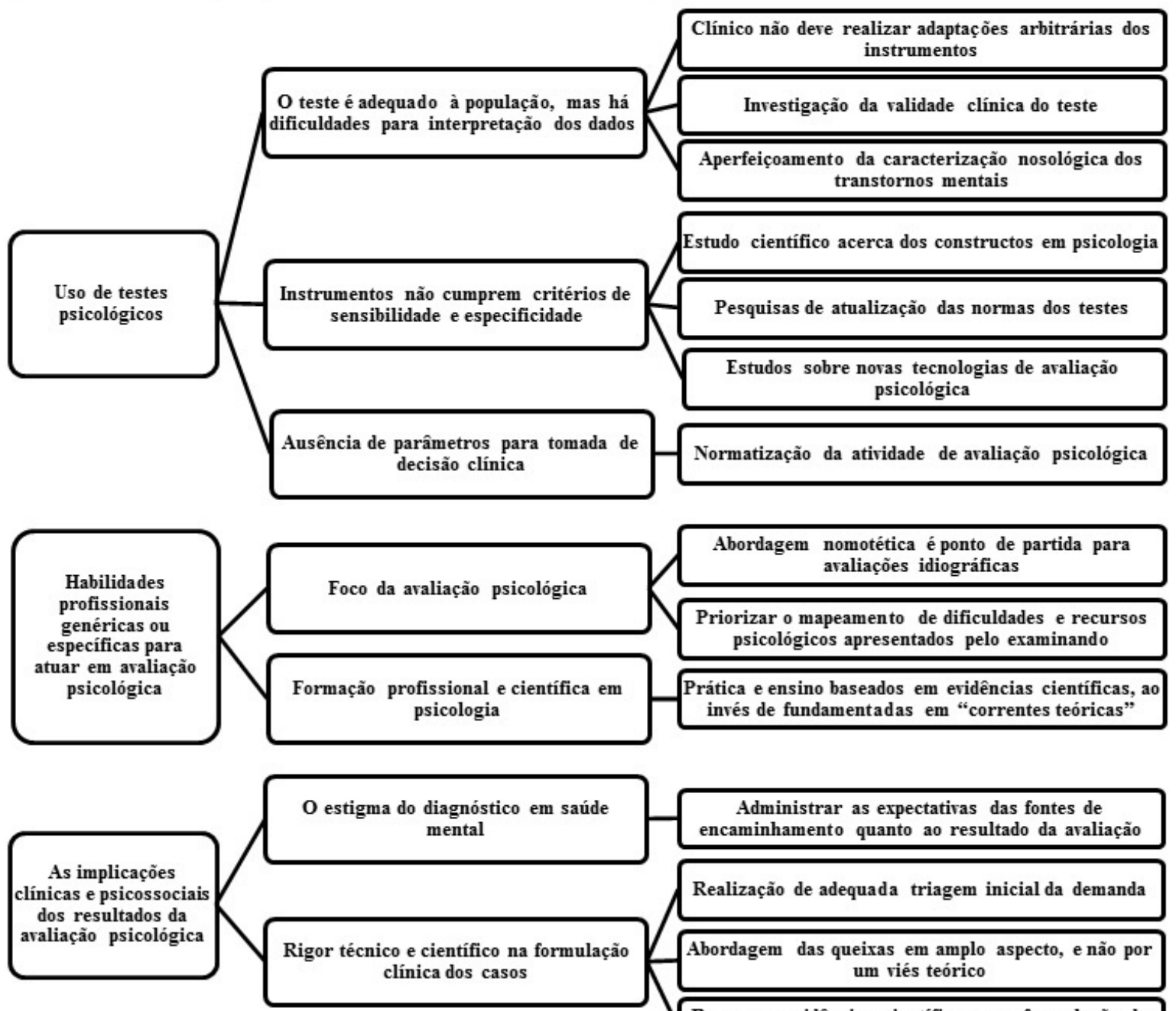
invés de fundamentadas em "correntes teóricas"

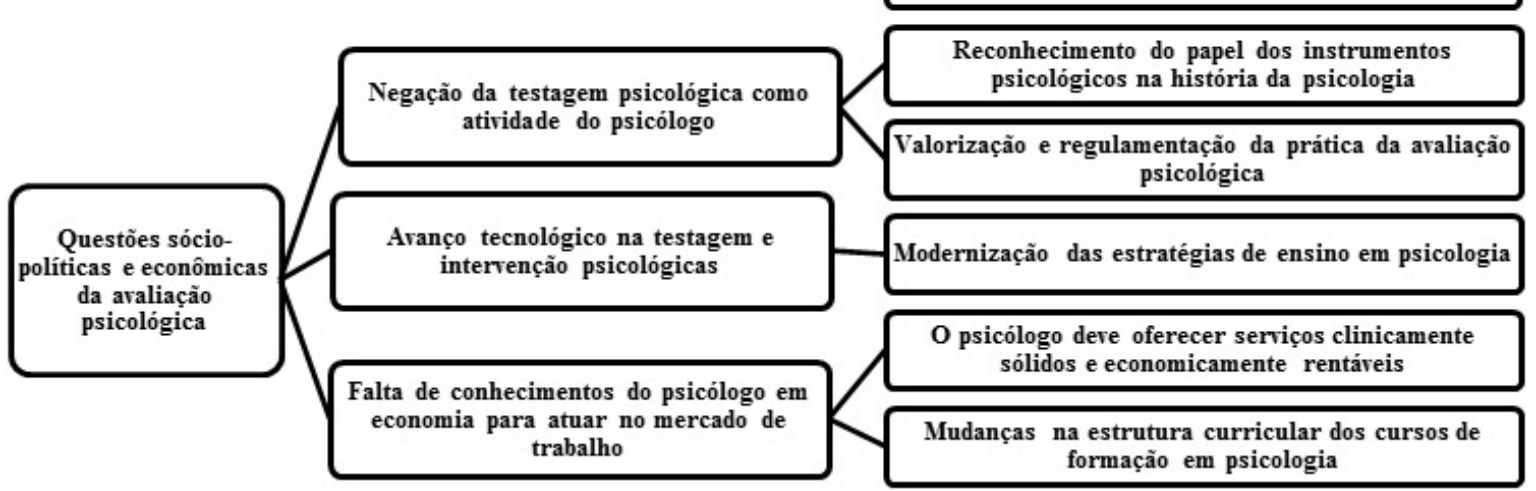

Figura 1. Temas e situações-problema da atuação em avaliação psicológica, com as respectivas linhas de pesquisas sugeridas no estudo.

\section{O Problema do Uso de Testes Psicológicos para Diferentes Contextos e Populações}

Um dos primeiros passos da testagem psicológica concerne à escolha do teste, que deve ser validado e padronizado em uma amostra que apresenta características que podem ser comparadas com as do cliente. Essas características podem variar como faixa etária, grau de escolaridade, gênero etc., ou seja, o examinando tem que apresentar características que são 
representadas pelos componentes da amostra. A escolha inadequada de teste critério leva a um resultado com confiabilidade questionável, e, nesses casos, não se pode inferir nem significados clínicos, nem psicométricos sobre o cliente (Leite, 2011). Esta questão foi analisada no presente estudo, em destaque para o contexto de avaliação psicológica para o trânsito. Neste, a interpretação dos dados da avaliação é feita, segundo os parâmetros dos testes, com base na escolaridade do indivíduo. Entretanto, existem situações que caracterizam polêmicas. É possível que clientes estejam afastados a muitos anos da escola e apresentem um baixo desempenho geral. Para a psicóloga P1, "Quando o examinando demonstra grandes diferenças no resultado do teste com a população de referência levantamos hipóteses sobre fatores externos influenciando os dados, como sono ... e até quantidade de anos de afastamento da escola... que está longe da escola". A falta de prática e treino das habilidades testadas durante o afastamento da escola pode contribuir para esses resultados. Ou seja, nestes casos, parece ser que o instrumento não é sensível nem específico à identificação de alterações cognitivas e diferenças entre grupos (Howieson \& Lezak, 2014).

Uma forma de manejar estes problemas seria a verificação da adequabilidade do instrumento para o caso, e ter como foco os objetivos do uso dos testes (Groth-Marnart \& Wright, 2016). Esta solução exige, por outro lado, que sejam feitas pesquisas por instrumentos que atendam tais especificidades. Nakano, Sampaio, e Silva (2011) já haviam mencionado a necessidade de desenvolver instrumentos para a realidade da psicologia do trânsito de forma a que os candidatos à renovação de carteira de habilitação não sejam avaliados pelos mesmos parâmetros que candidatos à primeira carteira, como ocorre atualmente.

Em estudo que comparou motoristas infratores, não infratores e envolvidos em acidentes (Nakano \& Sampaio, 2016) não foi possível verificar diferenças de desempenho cognitivo (inteligência e atenção) em função do perfil de comportamento no trânsito. A variável escolaridade foi, por outro lado, determinante nas diferenças identificadas entre os motoristas. Estes dados salientam a importância da variável "escolaridade" no processo de investigação psicológica no trânsito. As informações indicam que deveria haver uma forma mais eficaz de avaliar os candidatos que, em virtude da falta de prática, não mais apresentam o mesmo nível de habilidades que a amostra de padronização de mesma escolaridade e considerar o processo de avaliação como um todo e a interpretação adequada dos escores obtidos dentro de um contexto.

Não há uma sistematização objetiva das habilidades que diferenciem os tipos de condutores de veículos, não é possível regulamentar métodos específicos de avaliação psicológica para cada tipo de público. A psicóloga P1 mencionou que "discutimos em equipe outras formas de avaliação para estes casos. Também participamos dos debates sobre a regulamentação dos procedimentos... Mas as decisões lá de cima [instâncias superiores de normatização da avaliação psicológica para o trânsito] são muito lentas".

O Conselho Federal de Psicologia (CFP, 2009) sugere que sejam observadas algumas habilidades mínimas, como capacidade de codificação e processamento de informações (atenção); tomada de decisão e traços de personalidade. O trabalho do psicólogo, portanto, é utilizar os protocolos de avaliação existentes de modo sensato e flexível, para que seja possível estabelecer uma predição do comportamento do condutor no contexto em que ele deverá atuar (Araújo, Malloy-Diniz, \& Rocha, 2009).

As observações sobre a flexibilidade do processo de avaliação também cabem ao contexto da neuropsicologia. A avaliação de crianças, por exemplo, requer instrumentos espe- 
cíficos e métodos de exame clínico que possam abranger a avaliação das funções cognitivas (atenção, memória, praxias, linguagem, percepção, visuo-construção e funções executivas) (Howieson \& Lezak, 2014) e aspectos psicoafetivos. Deste modo, é preciso lançar mão de recursos técnicos que nem sempre estão explícitos nos manuais. Por exemplo, ao falar sobre a validade das informações obtidas pelos clientes em entrevistas não-estruturadas, uma das psicólogas menciona que

Nós somos treinados para estar com o paciente, a ouvi-lo. Não se preocupe se aquilo que te falam é verdade ou mentira, a verdade é aquela trazida no nosso consultório. Então, seja como for, aquele relato veio de uma mãe que me trouxe a impressão dela dos fatos, ou seja, como as coisas aconteceram de acordo com o seu ponto de vista. (P2)

Este panorama da flexibilidade no processo de avaliação torna-se complexo quando falamos dos transtornos do neurodesenvolvimento. Os dados de testes psicológicos serão comparados com a amostra de padronização que não apresentava déficits cognitivos, comprometendo a qualidade do instrumento em termos de sensibilidade e especificidade para o contexto do desenvolvimento (Howieson \& Lezak, 2014). De outra forma, é possível que na comparação de parâmetros algumas variáveis interfiram na análise, como por exemplo, a presença de comorbidades psiquiátricas (Leite, 2011).

Uma temática comum identificada nas entrevistas com a profissional da psicologia, bem como durante as observações sistemáticas do contexto clínico foi a carência de instrumentos para avaliar determinadas categorias nosológicas recentemente descritas nos manuais (American Psychiatry Association [APA], 2014). Por exemplo, o diagnóstico da discalculia do desenvolvimento, que se refere às dificuldades crônicas de aprendizagem da matemática, é realizado mediante critérios comportamentais, ou seja, não envolve, necessariamente, a avaliação neuropsicológica. De toda forma a escolha dos testes segue uma diretriz. A psicóloga P2 mencionou que "a escolha dos testes sempre terá relação com a queixa". Assim, esta avaliação será importante para caracterizar domínios cognitivos preservados ou comprometidos, bem como servirá de linha de base (por exemplo, na avaliação da inteligência) para verificação de resultados de intervenção psicológica (Haase, Júlio-Costa, \& Santos, 2015).

Por fim, com relação à adaptação dos métodos de avaliação, durante o presente estudo verificou-se, através do relato da experiência, que os perfis de desempenho sugeridos pelos manuais dos testes muitas vezes falham em indicar os déficits cognitivos de populações clínicas, ou seja, os índices de sensibilidade são baixos. Uma das psicólogas mencionou: "Tivemos a situação que um determinado teste não indicava alterações da atenção. Todo mundo que fazia gabaritava [sic] o teste!" (P1). As informações dos perfis são insuficientes ou inconsistentes com os demais dados coletados, muito em função das variabilidades individuais (Gonçalves, Simões, \& Castro-Caldas, 2014).

Desta forma, verifica-se que as falhas na interpretação qualitativa do desempenho dos examinandos podem enviesar a probabilidade de obtenção de índices seguros que relacionem a medida com a condição ou fenômeno psicológico em estudo (Fonseca, Zimmermann, \& Kochhann, 2014), por isso deve-se estar atento ao processo de avaliação como um todo fazendo a interpretação desses índices dentro de um contexto para chegar a conclusões mais adequadas e confiáveis. A avaliação psicológica é diferente de testagem psicológica e muitas vezes elas são interpretadas como sinônimos, mesmo que o teste seja uma importante fonte 
de informações a avaliação é um processo mais amplo e flexível. Mesmo que a avaliação psicológica venha sendo reconhecida, existem problemas concernentes a sua prática e que em grande parte se justificam pela formação desqualificada e despreparo técnico e teórico dos psicólogos em sua atuação (Borsa, 2016).

\section{Habilidades Profissionais para Atuação em Avaliação Psicológica: O Especialista ou o Generalista?}

O contexto da avaliação psicológica requer do profissional conhecimento e habilidades de diferentes áreas da psicologia, bem como áreas afins, sem que ele perca a especialização das técnicas de testagem psicológica. Na abordagem destas questões, os estudantes participantes do estudo se questionaram sobre as direções na formação profissional. Como ser um técnico competente no uso dos testes psicológicos, sem perder o caráter idiográfico da avaliação?

Nas supervisões realizadas no estudo foi abordada uma aparente contradição, mencionada por Primi (2010). Na sua prática, o psicólogo busca comparar o escore do examinando em uma determinada habilidade com o obtido por uma amostra de padronização do teste. Para alguns indivíduos, esta avaliação é insuficiente para estabelecer inferências. Por exemplo, na análise das funções executivas, um examinando poderia se sair bem em um teste de flexibilidade cognitiva de modalidade visuoespacial, mas ser classificado abaixo da média em um teste que avalia a mesma habilidade, porém, na modalidade verbal. Assim como Primi (2010) e Groth-Marnart e Wright (2016) defenderam, esta aparente contradição é resolvida considerando que as conclusões obtidas mediante a abordagem nomotética são hipóteses, ou pontos de PARTIDA para avaliações subsequentes que considerem os aspectos individuais do examinando. No caso mencionado, é possível supor que funções cognitivas superiores estejam em déficit, porém, de modo específico, elas estão sofrendo a intermediação de processos perceptuais básicos, que comprometem o desempenho. Sendo assim, adotou-se uma abordagem integrativa, na qual este aspecto específico do examinando será melhor explorado em tarefas que fornecerão melhores indícios sobre esta interação entre funções cognitivas.

Os estudantes participantes da pesquisa identificaram na prática de formação em avaliação psicológica que a atuação abrangente do psicólogo diz respeito à capacidade de superar o modelo de intervenção baseado estritamente nas habilidades de detectar e caracterizar determinada disfunção cognitiva em favor de uma atuação voltada para identificar, também, forças cognitivas. Este comprometimento depende de um adequado levantamento de dados qualitativos, e não somente da verificação de um escore no teste. Neste sentido, em um dos relatórios do estágio a aluna descreve o quanto ficou positivamente surpreendida com a condução das entrevistas feitas pelas psicólogas aos candidatos do processo de obtenção de carteira de motorista: "A entrevista me impressionou muito, pois achei que deixou o processo todo muito mais 'pessoal', com mais contato com o examinado" (E1).

Assim como argumentaram Winograd, Jesus, e Uehara (2012), não basta verificar se o paciente está abaixo da média da população de referência para aquele domínio cognitivo. É preciso verificar o processo que o levou a um resultado específico em um teste psicológico, ou seja, o método que o examinando utilizou para desempenhar a tarefa. Uma das alunas do estágio, referindo-se ao acompanhamento que fez de um caso clínico em neuropsicologia, disse "Vi que a avaliação é mais do que analisar testes. O psicólogo deve saber lidar com familiares, escola e cuidar do conforto do paciente durante a testagem" (E2). 
Uma vez que a avaliação psicológica de qualidade depende tanto do conhecimento específico sobre as habilidades a serem avaliadas e o domínio de aspectos gerais que intercedem o processo, sugere-se que a atuação profissional pode ser bem-sucedida se ele conseguir transitar entre estes indicadores na execução da avaliação. Por exemplo, na avaliação das funções executivas, além dos aspectos cognitivos ditos "frios", como raciocínio, atenção, inibição; é preciso considerar outros elementos, ou as "funções executivas quentes", como a tomada de decisão, que são permeados por fatores da personalidade e processamento emocional (Fonseca et al., 2012). Nas discussões de casos clínicos os estudantes verificaram que, se apenas um dos componentes das funções executivas for avaliado, ou se estes componentes forem identificados por métodos limitados, há risco de assumirmos erros diagnósticos do tipo falsos-positivos ou falsos-negativos, quando apenas um dos componentes das funções executivas estiver comprometido, ou se for negligenciada a sua natureza multifatorial.

A experiência do estágio levou os estudantes a refletir que as atividades do psicólogo realmente transitam entre o formato específico e generalista. Por exemplo, o psicólogo deve utilizar conhecimentos específicos da psicologia cognitiva para que possa avaliar os processos atencionais, memória e orientação visuoespacial. Adicionalmente, ele deve ter amplo conhecimento das teorias e técnicas sobre tomada de decisão, traços de personalidade e outros aspectos para obter registros de amostras do comportamento necessárias para julgar se o candidato está apto ou não para conduzir veículo automotor (CFP, 2009). A vivência desta atuação fez com que os alunos quebrassem paradigmas na formação profissional. Um dos estudantes mencionou "Eu achei que [o estágio] seria [aprendizagem apenas sobre] só teste. No final me surpreendi com tantas tarefas que o psicólogo faz" (E3).

Mesmo que os currículos de formação em psicologia sejam determinados por variáveis internas e externas à instituição de ensino, os componentes da matriz deveriam expressar a unidade da relação entre conteúdos generalistas e específicos (Seixas, Coelho-Lima, Silva, \& Yamamoto, 2013). Mais do que isto, as práticas profissionais e de ensino da profissão, apoiadas em "tradições teóricas" em psicologia, deveriam ser substituídas por conhecimentos sobre a psicologia humana que fossem fundamentados cientificamente. A "orientação teórica" não deveria sobrepujar a prática baseada em evidências (Melchert, 2016).

Ainda sobre o treino de habilidades profissionais, há uma questão ética na qual o profissional deve lidar: as consequências psicossociais do diagnóstico. Muitas vezes o diagnóstico em saúde mental é carregado de estigma, dificultando a busca por tratamento pelo paciente. O preconceito acerca dos transtornos psicológicos promove a desconexão entre a eficácia do tratamento e a busca de ajuda pelo paciente (Corrigan, Druss, \& Perlick, 2014).

O julgamento clínico em um processo de avaliação é passível de erros, seja por falhas na coleta de dados, na síntese dos dados, na relação entre experiência clínica versus dados estatísticos e na decisão sobre o que apresentar no relatório ou laudo psicológico. Para que estes aspectos não afetem negativamente os desfechos da avaliação, é ético e prudente que o profissional tenha conhecimento e adote estratégias para limitar estes erros. No caso das entrevistas com examinandos, por exemplo, o profissional deve combinar as respostas do cliente com os dados observados para levantar hipóteses sobre fenômenos psicológicos (Amorim \& Cardoso, 2015). Estas hipóteses devem ser testadas em seguida, para que seja possível refutá-las, aceitá-las ou modificá-las (Groth-Marnart \& Wright, 2016). No contexto da entrevista inicial na avaliação para o trânsito, por exemplo, a profissional P1 relatou aos alunos do estágio que os 
candidatos frequentemente emitem respostas que consideram satisfazer às expectativas do examinador quanto aos comportamentos esperados no trânsito. Resta ao psicólogo identificar estratégias para obter dados que mais se aproximam do fenômeno que se quer medir.

Ainda no contexto do trânsito, os estagiários observaram na prática que o público atendido é muito heterogêneo em termos idade, nível sociocultural, escolaridade e outras características. Isto demanda perícia em termos da coleta e interpretação dos dados. Em relatório da entrevista com a psicóloga a estudante E2 descreve que

A avaliação dos candidatos a motoristas é feita por um longo processo. A primeira etapa trata-se de uma entrevista psicológica de suma importância que visa avaliar alguns aspectos do candidato como o consumo de bebida alcoólica ou outras drogas, relacionamento familiar, temperamento do candidato, estado de saúde, características pessoais, entre outras. Depois, se o candidato for considerado apto pela psicóloga, entramos na etapa de aplicação de testes. Na sessão de devolutiva com o candidato a psicóloga aponta a aptidão ou não do candidato a tirar carteira. Ao final, ele deve assinar um termo alegando que entendeu o resultado da devolutiva, e não apresenta dúvidas. (E2)

Neste contexto, o profissional deve conhecer material teórico e empírico acerca da população atendida, de modo a obter estimativas corretas acerca de seus julgamentos (Groth-Marnart \& Wright, 2016). Adicionalmente, o profissional deve estar familiarizado com a literatura atual na área, por exemplo, sobre o avanço tecnológico no campo da condução de veículos automotores, e o impacto desta tecnologia no processamento de informações no trânsito.

Durante a realização do estágio os estudantes verificaram que a avaliação psicológica é um processo dinâmico de tomada de decisão, em qualquer contexto. Faz parte do desenvolvimento de competências profissionais nesta área o aprendizado de que, para limitar os erros na tomada de decisão, o psicólogo deve se ater para não assumir vieses teóricos, considerar e testar todas as hipóteses e ser criterioso na coleta de dados e na maneira de integrar os dados (Suhr, 2012).

\section{A Atuação em Avaliação Psicológica e as Questões Sócio-Políticas e Econômicas da Profissão}

A experiência de estágio em dois contextos de avaliação psicológica favoreceu a discussão de aspectos institucionais da psicologia como profissão. Em primeiro lugar os estudantes constataram o importante papel da avaliação na prática clínica. Por meio de uma adequada avaliação, é possível obter uma compreensão profunda e complexa do sujeito. Entretanto, foi discutida a situação na qual por muitas vezes a avaliação é negligenciada ou mal compreendida como promotora de estigma em saúde mental. Esta abordagem pré-concebida da avaliação psicológica, mais precisamente do uso de testes, afeta negativamente a psicologia como profissão. Ao negar a avaliação como tarefa do psicólogo, por consequência nega-se um conjunto de conhecimentos básicos da avaliação (e por que não, da origem da Psicologia), que foram historicamente constituídos, como os relativos ao desenvolvimento humano, processos cognitivos e aprendizagem (Woody \& Viney, 2017).

Segundo Tavares (2010) precisamos resgatar o movimento de regulamentação do uso de testes pela Psicologia, não meramente por uma defesa da profissão, mas porque a Psicologia é comprometida com padrões éticos e técnicos que asseguram o adequado uso de instru- 
mentos para realização de inferências sobre fenômenos psicológicos. Dito de outra forma, "o desenvolvimento da avaliação psicológica sempre foi um empreendimento cientificamente fundamentado, intimamente atrelado ao surgimento e à consolidação da Psicologia como ciência e profissão" (p. 34).

Os casos discutidos na experiência do estágio, observados nos contextos da neuropsicologia e psicologia do trânsito foram relevantes para reforçar a conclusão de que a prática da avaliação psicológica deve ser valorizada, mas também regulamentada. Esta medida serve para evitar excessos e equívocos de profissionais mal preparados ou tendenciosos por interesses pessoais ou de organizações. A análise da regulamentação da matéria permite dizer que a psicologia é a profissão que mantém uma estrutura de ensino e formação técnica adequada para atender às necessidades de avaliação psicológica da sociedade (Tavares, 2010). No relatório final de estágio, ao descrever a entrevista que realizou com a psicóloga do trânsito a aluna E1 mencionou que

Muitas clínicas fazem o uso inadequado destes testes, não levam a sério alguns critérios como idade, se o paciente apresenta alguma deficiência, não estudam a respeito da validade dos testes, quais seriam os mais atuais, os melhores para se aplicar em cada situação. Além disso, a maioria das clínicas também não realizam a entrevista inicial, onde é possivel entender o comportamento e diversos traços do candidato, o que ajuda não só a entender se o candidato está ou não apto a tirar carteira, como também na escolha dos testes a serem com ele utilizados. (E1)

A polêmica do uso exclusivo dos testes pelos profissionais da psicologia foi tratada no presente estudo mediante o relato da experiência dos estagiários. Por ser um campo de interface com outras áreas de conhecimento, a avaliação neuropsicológica tem sido considerada como campo de trabalho não exclusivo do psicólogo. Como consequência, os testes que são desenvolvidos a partir desta fundamentação teórica (interação entre estruturas/funções neurais e comportamento) são considerados por profissionais não-psicólogos como de uso não exclusivo do psicólogo (Haase et al., 2012).

Os estudantes participantes deste estudo verificaram que a avaliação psicológica vive um impasse como ciência e profissão. Isto porque o avanço tecnológico e científico aproximou a trajetória da Psicologia com os estudos psicobiológicos que indicam a relação entre funcionamento neural e manifestações cognitivas e comportamentais. Em função de estas temáticas serem estudadas por outras áreas do conhecimento, os instrumentos e técnicas desenvolvidos desde então foram questionados como não exclusivos do psicólogo. Como consequência, o psicólogo se vê despreparado quanto aos conhecimentos de gerência de negócios para lidar com a concorrência no campo de atuação.

A Psicologia não pode abdicar dos conhecimentos acumulados, nem retroceder para explicações reducionistas (estritamente sociológicas ou culturais) com o intuito de defesa da profissão. Por outro lado, não é possível negligenciar que a Psicologia detém uma história de desenvolvimento científico atrelada à origem dos testes psicológicos (Woody \& Viney, 2017), e que possui um sistema de regulação da formação profissional e do uso de instrumentos psicológicos para oferecer serviços com competência e ética (Tavares, 2010). Talvez, enquanto estes princípios não forem regulamentados no campo interdisciplinar, a Psicologia ainda oferece melhores requisitos para atendimento da demanda por avaliação psicológica. 
O avanço nas tecnologias de avaliação psicológica promete revolucionar, também, o campo de atuação do psicólogo. Um dos alunos ressaltou em seu relatório de estágio que, ao final da experiência pôde concluir que "para ser um profissional de avaliação psicológica é necessária atualização constante, estudos variados nas áreas afins e acompanhamento da evolução de teorias e métodos de avaliação" (E3). O uso de computadores na avaliação psicológica não é novidade, mas o aumento da disponibilidade destes dispositivos eletrônicos, conectados em rede mundial de computadores para avaliação de pacientes simplificou a administração e pontuação dos testes (Kane \& Parsons, 2017). Há ainda a promessa feita por aplicativos de celular de avaliar e treinar processos cognitivos e comportamentais no estilo "faça você mesmo". Existem recursos disponíveis para o manejo de psicopatologias, mas não há controle ou qualquer avaliação profissional ou científica sobre os dispositivos (Lipczynska, 2016). Neste âmbito, é imperativo a modernização das estratégias de ensino, com incorporação das evidências científicas recentes sobre a testagem computadorizada e valorização da ciência psicológica. Estas novas tecnologias devem ser discutidas quanto às suas vantagens, limitações e custos, sem perder de vista o debate sobre a probabilidade de substituição do trabalho do psicólogo pelas interpretações automáticas das respostas dos examinandos nos testes informatizados (Kane \& Parsons, 2017).

\section{Considerações Finais}

As concepções que os profissionais e estagiários dos campos de atuação da neuropsicologia e psicologia do trânsito apresentaram no presente estudo revelam aspectos importantes acerca desta experiência por eles vivenciada. Eles indicaram seus posicionamentos acerca dos conhecimentos e das habilidades necessários para atuar nestes domínios da prática. As observações realizadas por eles sugerem reflexões sobre aspectos teóricos e práticos que poderão ser melhor investigados em pesquisas futuras.

Dentre os pontos de vista apresentados, podemos destacar os seguintes temas dentro da categoria "prática profissional": 1) Prática baseada em evidências científicas; 2) Abordagem em amplo espectro do motivo da avaliação, e manejo das expectativas das fontes de encaminhamento; 3) Os parâmetros de análise dos desempenhos nos testes e a abrangência das inferências acerca dos fenômenos psicológicos; 4) O mapeamento de dificuldades e recursos psicológicos apresentados pelo examinando e a relação com o objetivo de formulação diagnóstica dos casos.

Quanto à ciência psicológica, podem ser destacados os seguintes tópicos na argumentação dos profissionais e estudantes: 5) A caracterização nosológica dos transtornos mentais; 6) Investigação da validade clínica dos testes; 7) Estudo científico dos constructos em psicologia; 8) Pesquisas sobre novas tecnologias de avaliação psicológica.

O presente estudo apresenta limitações quanto ao alcance das inferências realizadas. No relato de experiência do estágio realizado pelo estudante é provável que algumas informações tenham sido registradas de modo incompleto, ou selecionadas para constar nos relatórios, enquanto outras não (Shaughnessy, Zechmeister, \& Zechmeister, 2012). De toda forma, buscou-se o cumprimento do método científico no qual as proposições foram organizadas de forma lógica e obtidas de variadas fontes de modo a descrever fatos e relações entre elementos de modo confiável. 
O exercício de interface entre teoria e a prática profissional possibilita o desenvolvimento de técnicas de intervenção mais eficazes, ao mesmo tempo em que são inseridas no campo de pesquisa as demandas originadas do contato com a comunidade que necessita de avaliação psicológica. Este debate sobre os pressupostos teóricos e metodológicos, questões éticas e o contexto sócio-político de inserção do psicólogo na avaliação psicológica deve ultrapassar o ambiente acadêmico, e perdurar durante toda a prática profissional.

\section{Referências}

American Psychiatry Association. (2014). Manual diagnóstico e estatístico de transtornos mentais DSM-5. (M. I. C. Nascimento et al. Trad.). Porto Alegre: Artmed.

Amorim, M. C., \& Cardoso, H. F. (2015). Entrevista psicológica na avaliação pericial em Psicologia do Trânsito: Uma análise crítica. Psicologia Revista, 24(1), 107-120. Disponível em https://revistas. pucsp.br/index.php/psicorevista/article/view/24231

Araújo, M. M., Malloy-Diniz, L. F., \& Rocha, F. L. (2009). Impulsividade e acidentes de trânsito. Revista de Psiquiatria Clínica, 36(2), 60-68. doi: http://dx.doi.org/10.1590/ S0101-60832009000200004

Borsa, J. C. (2016). Considerações sobre a formação e a prática em avaliação psicológica no Brasil. Temas em Psicologia, 24(1), 131-143. doi: http://dx.doi.org/10.9788/TP2016.1-09

Brown, T. G., Ouimet, M. C., Eldeb, M., Tremblay, J., Vingilis, E., Nadeau, L., Pruessner, J., \& Bechara, A. (2017). The effect of age on the personality and cognitive characteristics of three distinct risky driving offender groups. Personality and Individual Differences, 113(15), 48-56. doi: https://doi.org/10.1016/j.paid.2017.03.007

Casas, R. N., Calamia, M., \& Tranel, D. (2018). A global perspective on neuropsychological assessment. In S. G. Hofmann (Ed.). Clinical psychology, a global perspective (pp. 81-98). Hoboken, NJ: John Wiley \& Sons Ltda.

Castro, P. F. (2013). Caracterização do ensino de avaliação psicológica no Estado de São Paulo. Boletim de Psicologia, 63(138), 81-102. Disponível em http://pepsic.bvsalud.org/ pdf/bolpsi/v63n138/v63n138a08.pdf

Cohen, R. J., Swerdlik, M. E., \&Sturman, E. D. (2014). Testagem e avaliação psicológica: Introdução a testes e medidas (8a ed.) (Maria Cristina G. Monteiro, Trad.). Porto Alegre: AMGH Editora.

Conselho Federal de Psicologia. (2009). Resolução CFP no 007/2009, institui normas e procedimentos para a avaliação psicológica no contexto do Trânsito. Disponível em https://site.cfp.org.br/wp-content/uploads/2009/08/resolucao2009_07.pdf

Conselho Nacional de Trânsito. (2012). Resolução CONTRAN n. 425, de 27 de novembro de 2012. Dispõe sobre o exame de aptidão física e mental, a avaliação psicológica e o credenciamento das entidades públicas e privadas de que tratam o art. 147, I e $\S \S 1$ o a 4o e o art. 148 do Código de Trânsito Brasileiro. Brasília, DF. Disponível em http://www. denatran.gov.br/download/Resolucoes/(Resolu\%C3\%A7\%C3\%A30\%20425.-1).pdf

Corrigan, P. W., Druss, B. G., \& Perlick, D. A. (2014). The impact of mental illness stigma on seeking and participating in mental health care. Psychological Science in the Public Interest, 15(2), 37-70. doi: http://dx.doi.org/10.1177/1529100614531398

Eisman, E. J., \& Nordal, K. (2017). The implications for health care: Commentary on the special section on teaching, training, and supervision in personality and psycholocial assessment. 
Journal of Personality Assessment, 99(2), 186-188, doi: https://doi.org/10.1080/002238 91.2016.1226176

Fonseca, R. P., Zimmermann, N., \& Kochhann, R. (2014). Avaliação neuropsicológica: Bases para a interpretação quantitativa e qualitativa de desempenho. In F. H. Santos et al. (Orgs.), Neuropsicologia hoje (2a ed., pp. 106-114). Porto Alegre: Artmed.

Gonçalves, M. A., Simões, M. R., \& Castro-Caldas, A. (2014). Sugestões para interpretação dos desempenhos do manual da WAIS-IIII falham na identificação de defeitos cognitivos num grupo de doentes com tumores cerebrais. Anais do IX Congresso Iberoamericano de Psicologia e 2o Congresso da Ordem dos Psicólogos Portugueses, Lisboa, de 9 a 13 de setembro de 2014.

Groth-Marnat, G., \& Wright, A. J. (2016). Handbook of psychological assessment (6a ed.). Hoboken, NJ: John Wiley \& Sons.

Haase, V. G. et al. (2012). Neuropsicologia como ciência interdisciplinar: consenso da comunidade brasileira de pesquisadores/clínicos em Neuropsicologia. Revista Neuropsicologia Latinoamericana, 4(4), 1-8. Disponível em http://pepsic.bvsalud.org/ scielo.php?script=sci_arttext\&pid=\$2075-94792012000400001

Haase, V. G., Júlio-Costa, A., \& Santos, F. H. (2015). Discalculia do desenvolvimento. In F. H. Santos et al. (Orgs.), Neuropsicologia hoje (2a ed., pp. 160-168). Porto Alegre: Artmed.

Howieson, D. B., \& Lezak, M. (2014). A avaliação neuropsicológica. In S. C. Yudofsky, \& R. E. Hales (Orgs.), Fundamentos de neuropsiquiatria e ciências do comportamento (2a ed., pp. 41-65). Porto Alegre: Artmed.

Kane, R. L., \& Parsons, T. D. (2017). Introduction to neuropsychologyand technology. In R. L. Kane, \& T. D. Parsons (Eds.). The role of technology in clinical neuropsychology (pp. 3-26). New York, NY: Oxford University Press.

Leite, O. A. (2011). A medida no exame psicológico: Reflexões sobre o significado clínico da medida. In Conselho Federal de Psicologia. Ano da avaliação psicológica - Textos geradores (pp. 29-36). Brasília: Conselho Federal de Psicologia.

Lipczynska, S. (2016). The health apps on your smartphone: Science or snake oil? Journal of Mental Health, 25(5), 385-386. doi: http://dx.doi.org/10.3109/09638237.2016.1167859

Melchert, T. P. (2016). Leaving behind our preparadigmatic past: Professional psychology as a unified clinical science. American Psychologist, 71(6), 486-496. doi: http://dx.doi. org/10.1037/a0040227

Ministério da Educação (2011). Resolução CNE/CES 5/2011. Diário Oficial da União, Brasília, 16 de março de 2011 - Seção 1 - p. 19, institui as Diretrizes Curriculares Nacionais para os cursos de graduação em Psicologia, estabelecendo normas para o projeto pedagógico complementar para a Formação de Professores de Psicologia. Disponível em http://portal. mec.gov.br/index.php?option=com_docman\&view=download\&alias=7692-rces005-11pdf\&Itemid=30192

Nakano, T. C., \& Sampaio, M. H. L. (2016). Desempenho em inteligência, atenção concentrada e personalidade de diferentes grupos de motoristas. Psico-USF, 21(1), 147-161. doi: http:// dx.doi.org/10.1590/1413-82712016210113

Nakano, T. C., Sampaio, M. H. L., \& Silva, A. B. (2011). Atenção e inteligência em candidatos à primeira carteira nacional de habilitação. Boletim de Psicologia, LXI(134), 63-78. Disponível em http://pepsic.bvsalud.org/pdf/bolpsi/v61n134/v61n134a06.pdf 
Noronha, A. P. P., Castro, N. R., Ottati, F., Barros, M. V. C., \& Santana, P. R. (2013). Conteúdos e metodologias de ensino de avaliação psicológica: Um estudo com professores. Revista Paidéia, 23(54), 129-139. doi: http://dx.doi.org/10.1590/1982-43272354201315

Nunes, M. F. O., Muniz, M., Reppold, C. T., Faiad, C., Bueno, J. M. H., \& Noronha, A. P. P. (2012). Diretrizes para o ensino de avaliação psicológica. Avaliação Psicológica, 11(2), 309-316. Disponível em http://pepsic.bvsalud.org/pdf/avp/v11n2/v11n2a16.pdf

Primi, R. (2010). Avaliação psicológica no Brasil: Fundamentos, situação atual e direções para o futuro. Psicologia, Teoria e Pesquisa, 26(especial), 25-35. doi: http://dx.doi.org/10.1590/ S0102-37722010000500003

Seixas, P. S., Coelho-Lima, F., Silva, S. G., \& Yamamoto, O. H. (2013). Projeto pedagógico de curso e formação do psicólogo: Uma proposta de análise. Revista Semestral da Associação Brasileira de Psicologia Escolar e Educacional, 17(1), 113-122. Disponível em http://www. scielo.br/pdf/pee/v17n1/a12v17n1.pdf

Shaughnessy, J. J., Zechmeister, E. B., \& Zechmeister, J. S. (2012). Metodologia de pesquisa em Psicologia (9a ed.) (R. C. Costa, Trad.). Porto Alegre: AMGH Editora.

Suhr, J. A. (2012). Psychological assessment: A problem-solving approach. New York, NY: The Guilford Press.

Tavares, M. (2010). Da ordem social da regulamentação da Avaliação Psicológica e do uso dos testes. In Conselho Federal de Psicologia. Avaliação psicológica: Diretrizes na regulamentação da profissão (pp. 31-56). Brasília: Conselho Federal de Psicologia.

White, M. J., Young, R. McD., \& Rakotonirainy, S. (2012). Executive function development and stress effects on driving performance: Preliminary findings from a young adult sample. In M. Sullman, \& L. Dorn (Eds.), Advances in traffic psychology. New York, NY: Taylor \& Francis Group.

Winograd, M., Jesus, M. V. M., \& Uehara, E. (2012). Aspectos qualitativos na prática da avaliação neuropsicológica. Ciências \& Cognição, 17(2), 2-13. Disponível em http://pepsic. bvsalud.org/pdf/cc/v17n2/v17n2a02.pdf

Wit, P. A. J. M., Souza, C. Z., \& Cruz, R. M. (2016). Improving accident statistics and expanding the role of traffic psychologists in Brazil. Psicologia: Ciência e Profissão, 36(4), 816-830. doi: http://dx.doi.org/10.1590/1982-3703002382016

Woody, W. D., \& Viney, W. (2017). A history of psychology: The emergence of Science and applications (6a ed.). New York, NY: Taylor \& Francis.

Recebido: 05/05/2017

Última revisão: 12/04/2018

Aceite final: 18/04/2018

\section{Sobre as autoras:}

Jeanny Joana Rodrigues Alves de Santana - Docente do Instituto de Psicologia da Universidade Federal de Uberlândia, MG (UFU). Psicóloga pela UFU. Doutora e mestre em Psicobiologia pela Faculdade de Filosofia, Ciências e Letras de Ribeirão Preto, SP (USP/RP). Especialização e aprimoramento em Psicologia da Saúde no Hospital de Base da Faculdade de Medicina de São José do Rio Preto. E-mail: jeannysantana@yahoo.com.br

Marina Celestino Soares - Graduanda em Psicologia pela Faculdade Pitágoras, Uberlândia, MG. Pesquisadora em Neuropsicologia e Avaliação Psicológica. E-mail: marinacelestinosoares@gmail.com 